\title{
Detection and Localization of Tooth Breakage Fault on Wind Turbine Planetary Gear System considering Gear Manufacturing Errors
}

\author{
Y. Gui, ${ }^{1,2}$ Q. K. Han, ${ }^{1}$ Z. Li, ${ }^{1}$ and F. L. Chu ${ }^{1}$ \\ ${ }^{1}$ State Key Laboratory of Tribology, Department of Mechanical Engineering, Tsinghua University, Beijing 100084, China \\ ${ }^{2}$ Department of Mechanical Engineering, Academy of Armored Force Engineering, Beijing 100072, China \\ Correspondence should be addressed to F. L.Chu; chufl@mail.tsinghua.edu.cn
}

Received 18 May 2014; Revised 23 July 2014; Accepted 27 July 2014; Published 19 August 2014

Academic Editor: Ahmet S. Yigit

Copyright @ 2014 Y. Gui et al. This is an open access article distributed under the Creative Commons Attribution License, which permits unrestricted use, distribution, and reproduction in any medium, provided the original work is properly cited.

\begin{abstract}
Sidebands of vibration spectrum are sensitive to the fault degree and have been proved to be useful for tooth fault detection and localization. However, the amplitude and frequency modulation due to manufacturing errors (which are inevitable in actual planetary gear system) lead to much more complex sidebands. Thus, in the paper, a lumped parameter model for a typical planetary gear system with various types of errors is established. In the model, the influences of tooth faults on time-varying mesh stiffness and tooth impact force are derived analytically. Numerical methods are then utilized to obtain the response spectra of the system with tooth faults with and without errors. Three system components (including sun, planet, and ring gears) with tooth faults are considered in the discussion, respectively. Through detailed comparisons of spectral sidebands, fault characteristic frequencies of the system are acquired. Dynamic experiments on a planetary gear-box test rig are carried out to verify the simulation results and these results are of great significances for the detection and localization of tooth faults in wind turbines.
\end{abstract}

\section{Introduction}

Planetary gear systems have been widely used in wind power systems because of the advantages of compact structure, large carrying capacity, and high transmission efficiency [1]. In recent years, the tooth faults that have occurred in planetary gear systems have brought numerous troubles to wind power plants [2-4]. Therefore, fault diagnosis of the planetary gear system is of great significant to the safe operation of wind turbine.

Tooth pitting, spalling, cracking, and breakage are some of the common fault modes that have occurred in wind turbine planetary gear systems. Detection and localization of those faults are of great significance. Vibration based diagnosis is one of the most effective and frequently used health monitoring technologies. In recent years, much attention has been paid on analyzing the vibration signal in time and frequency domains. To name a few, Lei et al. [5] extracted two diagnostic parameters based on the examination of vibration characteristics of a planetary gearbox in both time and frequency domains. Experiments revealed that the proposed diagnostic parameters performed better than other parameters. Lin and Zuo [6] introduced a method based on the Morlet wavelet time-frequency analysis. The method was found more effective in detection of tooth cracks than two other types of discrete wavelet transform. Liu et al. [7] carried out fault diagnosis based on the local mean decomposition (LMD) method. The method was suitable for obtaining instantaneous frequencies in frequency domains.

The above-mentioned studies are helpful for the fault detection of planetary gear system. In addition, the sidebands of vibration signals also attract the attention of many researchers, because the sidebands contain abundant information which is useful for the detection and localization of faults in planetary gear systems. McFadden and Smith [8] found that the modulation sidebands of the planetary gear system are typically asymmetric and the frequency 
with largest amplitude is the multiple of the carrier rotation frequency. Based on the research, McFadden [9] presented a method to calculate the time domain average of the vibration signal and the method for the separation of planet vibration and sun gear vibration is extracted. Feng and Zuo [10] deduced the relationship between fault characteristic frequencies and rotating frequencies of the components in a planetary gear system. The complicity of sidebands and fault characteristic frequencies were verified by simulations and experiments. Chaari et al. [11] modeled a planetary gear system which contained the fault modes of tooth pitting and cracking. The dynamic responses of the fault systems and a healthy gear system were compared in frequency domains. Chaari et al. [12] also studied the influence of tooth pitting and cracking on the sun gear vibration spectrum based on a planetary gear model. Sidebands were found to be induced because of amplitude modulation. Cheng et al. $[13,14]$ estimated the degree of gear pitting and cracking by comparing the dynamic response of a fault system with a health system. A model containing tooth spalling and cracking was developed by Jia and Howard [15]. His research indicated that the amplitude and phase modulation of the vibration signal could indicate the difference between tooth spalling and cracking effectively.

However, various gear manufacturing errors were not considered in the above-mentioned investigations. Typical gear errors, such as eccentricities, pitch-line run-out errors, tooth spacing, and indexing errors, are inevitable in actual planetary gear system. Ligata et al. [16] pointed out that the aforementioned errors of a planetary gear set might cause modulations to the sidebands of the system. Inalpolat and Kahraman [17] developed a nonlinear time-varying dynamic model to predict modulated sidebands of a planetary gear system, and both the numerical and experimental results showed that the sidebands were greatly changed due to the amplitude and frequency modulations caused by gear errors. As the gear errors can influence the sidebands significantly $[16,17]$, the fault characteristics might be greatly changed after considering the gear errors. Therefore, it is necessary to take the influence of errors into consideration to increase the accuracy and precision of the tooth faults diagnosis.

In this paper, a lumped parameter model of a typical planetary gear system is established. The influences of typical tooth faults on the time-varying mesh stiffness and tooth impact forces are derived analytically. Numerical methods are utilized to obtain the response spectra of the system with tooth faults with and without gear errors. Three system components (including sun, planet, and ring gears) with tooth faults are considered, respectively, in the discussion. Through detailed comparisons on spectral sidebands, fault characteristic frequencies of the system are acquired. Dynamic experiments on a planetary gear-box test rig are carried out to verify the simulation results.

\section{Dynamic Model of Planetary Gear System}

Figure 1 shows the schematic diagram of the dynamic model for a planetary gear system. The system composes of sun

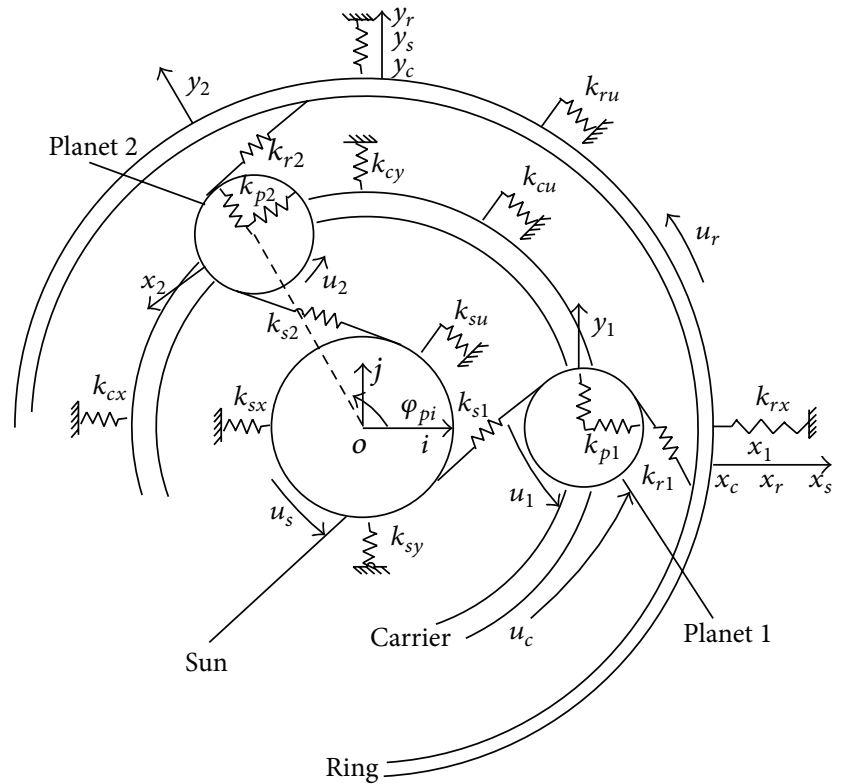

FIGURE 1: The dynamic model for a planetary gear system.

gear $(s)$, carrier $(c)$, ring $(r)$, and three planets $(p)$. Bearings are considered as linear springs and gear meshes are considered as linear springs acting on the lines of action. Each component has three degrees of freedom, transverse $(x)$, longitudinal $(y)$, and torsional degrees $(u)$.

The reference coordinate system rotates with the carrier and the origin of the coordinate coincides with the center of the carrier. The circumferential positions of planets are specified by the fixed angles $\varphi_{p_{i}}$ (Figure 2) measured relatively to the rotating coordinate system with $\varphi_{p_{1}}=0$.

The global equation of motion for the system can be simplified as $[18,19]$

$$
\begin{aligned}
\mathbf{M} \ddot{\mathbf{q}}+ & \Omega_{c} \mathbf{G} \dot{\mathbf{q}}+\left[\mathbf{K}_{b}+\mathbf{K}_{e}(t)-\Omega_{c}^{2} \mathbf{K}_{\Omega}\right] \mathbf{q} \\
& =\mathbf{T}(t)+\mathbf{F}_{W}(t)+\mathbf{F}_{e}(t),
\end{aligned}
$$

where $\mathbf{q}$ represents the vector of the degrees of freedom

$$
\begin{array}{r}
q=\left\{x_{c}, y_{c}, u_{c}, x_{r}, y_{r}, u_{r}, x_{s}, y_{s}, u_{s},\right. \\
\left.x_{1}, y_{1}, u_{1}, \ldots, x_{N}, y_{N}, u_{N}\right\}^{T} .
\end{array}
$$

$\Omega_{c}$ is the rotating speed of the carrier. Due to the unstable wind conditions, frequent low speed start and emergency brakes, the rotating speed is often nonstationary. However, if the concerned time period is small, the work condition of the wind turbine could be considered as quasi-stable [20]. Thus, the premise of constant rotating speed is used in this paper. 


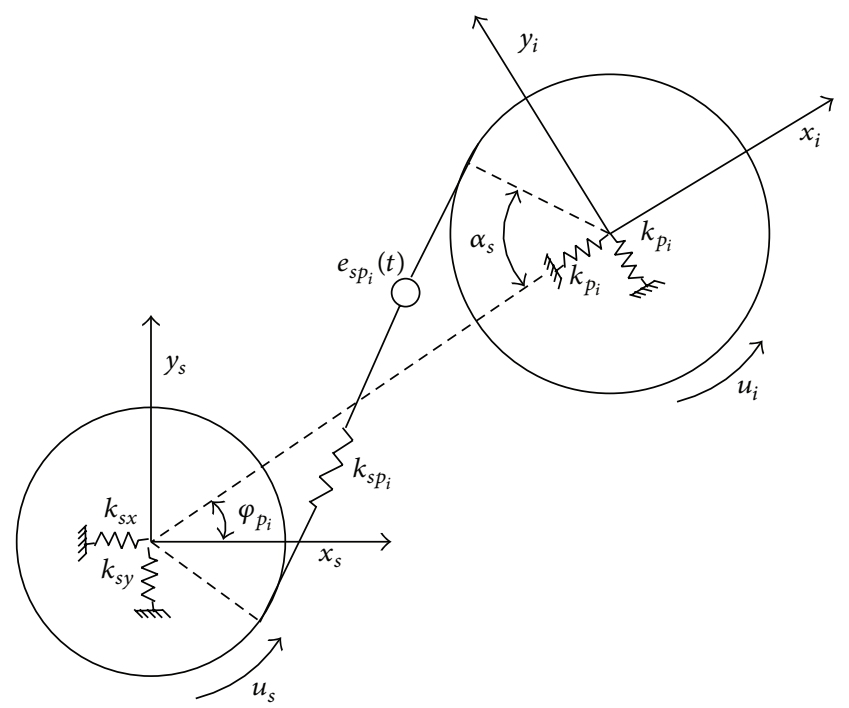

Figure 2: A sun-planet mesh.

M, $\mathbf{G}$ represent the mass and gyroscopic matrices, respectively, and are expressed by

$$
\begin{aligned}
& \mathbf{M}=\left[\begin{array}{cccccccc}
\mathbf{M}_{c} & 0 & 0 & 0 & 0 & 0 & \cdots & 0 \\
0 & \mathbf{M}_{r} & 0 & 0 & 0 & 0 & \cdots & 0 \\
0 & 0 & \mathbf{M}_{s} & 0 & 0 & 0 & \cdots & 0 \\
0 & 0 & 0 & \mathbf{M}_{1} & 0 & 0 & \cdots & 0 \\
0 & 0 & 0 & 0 & \mathbf{M}_{2} & 0 & \cdots & 0 \\
\vdots & \vdots & \vdots & \vdots & \vdots & \vdots & \vdots & 0 \\
0 & 0 & 0 & 0 & 0 & 0 & \cdots & \mathbf{M}_{N}
\end{array}\right] \\
& \mathbf{M}_{j}=\left[\begin{array}{ccc}
m_{j} & 0 & 0 \\
0 & m_{j} & 0 \\
0 & 0 & \frac{I_{j}}{r_{j}^{2}}
\end{array}\right], \quad j=c, r, s, 1, \ldots, N \\
& \mathbf{G}=\left[\begin{array}{cccccccc}
\mathbf{G}_{c} & 0 & 0 & 0 & 0 & 0 & \cdots & 0 \\
0 & \mathbf{G}_{r} & 0 & 0 & 0 & 0 & \cdots & 0 \\
0 & 0 & \mathbf{G}_{s} & 0 & 0 & 0 & \cdots & 0 \\
0 & 0 & 0 & \mathbf{G}_{1} & 0 & 0 & \cdots & 0 \\
0 & 0 & 0 & 0 & \mathbf{G}_{2} & 0 & \cdots & 0 \\
\vdots & \vdots & \vdots & \vdots & \vdots & \vdots & \vdots & 0 \\
0 & 0 & 0 & 0 & 0 & 0 & \cdots & \mathbf{G}_{N}
\end{array}\right], \\
& \mathbf{G}_{j}=\left[\begin{array}{ccc}
0 & -2 m_{j} & 0 \\
2 m_{j} & 0 & 0 \\
0 & 0 & 0
\end{array}\right], \quad j=c, r, s, 1, \ldots, N,
\end{aligned}
$$

where $m_{j}$ and $r_{j}$ are the mass and the base radius of part $j, I_{j}$ is the inertial moment of part $j$ with respect to its rotational axis.
$\mathbf{K}_{b}$ is the bearing stiffness matrices and are expressed by

$$
\begin{aligned}
\mathbf{K}_{b} & =\left[\begin{array}{cccccc}
\mathbf{K}_{c b} & 0 & 0 & 0 & \cdots & 0 \\
0 & \mathbf{K}_{r b} & 0 & 0 & \cdots & 0 \\
0 & 0 & \mathbf{K}_{s b} & 0 & \cdots & 0 \\
0 & 0 & 0 & 0 & \cdots & 0 \\
\vdots & \vdots & \vdots & \vdots & \vdots & 0 \\
0 & 0 & 0 & 0 & \cdots & 0
\end{array}\right], \\
\mathbf{K}_{j b} & =\left[\begin{array}{ccc}
k_{j x} & 0 & 0 \\
0 & k_{j y} & 0 \\
0 & 0 & k_{j u}
\end{array}\right],
\end{aligned}
$$

where $k_{j x}, k_{j y}, k_{j u}$ represent the bear supporting stiffness long the three degrees of freedom.

The centripetal stiffness matrix due to the carrier rotation is denoted by $\mathbf{K}_{\Omega}$. Consider

$$
\begin{aligned}
\mathbf{K}_{\Omega} & =\left[\begin{array}{ccccccc}
\mathbf{K}_{\Omega c} & 0 & 0 & 0 & 0 & \cdots & 0 \\
0 & \mathbf{K}_{\Omega r} & 0 & 0 & 0 & \cdots & 0 \\
0 & 0 & \mathbf{K}_{\Omega s} & 0 & 0 & \cdots & 0 \\
0 & 0 & 0 & \mathbf{K}_{\Omega 1} & 0 & \cdots & 0 \\
0 & 0 & 0 & 0 & \mathbf{K}_{\Omega 2} & \cdots & 0 \\
\vdots & \vdots & \vdots & \vdots & \vdots & \vdots & 0 \\
0 & 0 & 0 & 0 & 0 & 0 & \mathbf{K}_{\Omega N}
\end{array}\right], \\
\mathbf{K}_{\Omega j} & =\left[\begin{array}{ccc}
m_{j} & 0 & 0 \\
0 & m_{j} & 0 \\
0 & 0 & 0
\end{array}\right], \quad j=c, r, s, 1, \ldots, N .
\end{aligned}
$$

The time-varying mesh stiffness matrix is represented by $\mathbf{K}_{e}(t)$. The matrices are expressed as

$$
\mathbf{K}_{e}(t)=\left[\begin{array}{ccccccc}
\sum \mathbf{K}_{c 1}^{i} & 0 & 0 & \mathbf{K}_{c 2}^{1} & \mathbf{K}_{c 2}^{2} & \cdots & \mathbf{K}_{c 2}^{N} \\
0 & \sum \mathbf{K}_{r 1}^{i} & 0 & \mathbf{K}_{r 2}^{1} & \mathbf{K}_{r 2}^{2} & \cdots & \mathbf{K}_{r 2}^{N} \\
0 & 0 & \sum \mathbf{K}_{s 1}^{i} & \mathbf{K}_{s 2}^{1} & \mathbf{K}_{s 2}^{2} & \cdots & \mathbf{K}_{s 2}^{N} \\
\mathbf{K}_{c 2}^{1} & \mathbf{K}_{r 2}^{1} & \mathbf{K}_{s 2}^{1} & \mathbf{K}^{1} & 0 & \cdots & 0 \\
\mathbf{K}_{c 2}^{2} & \mathbf{K}_{r 2}^{2} & \mathbf{K}_{s 2}^{2} & 0 & \mathbf{K}^{2} & \cdots & 0 \\
\vdots & \vdots & \vdots & \vdots & \vdots & \vdots & 0 \\
\mathbf{K}_{c 2}^{N} & \mathbf{K}_{r 2}^{N} & \mathbf{K}_{s 2}^{N} & 0 & 0 & \cdots & \mathbf{K}^{N}
\end{array}\right],
$$


where

$$
\begin{aligned}
& \mathbf{K}_{r 1}^{i}=k_{r p_{i}}(t)\left[\begin{array}{ccc}
\sin ^{2} \varphi_{r p_{i}} & -\cos \varphi_{r p_{i}} \cos \alpha_{r} & \sin \varphi_{r p_{i}} \\
-\cos \varphi_{r p_{i}} \cos \varphi_{r} & \cos ^{2} \varphi_{r p_{i}} & \cos \varphi_{r p_{i}} \\
\sin \varphi_{r p_{i}} & \cos \varphi_{r p_{i}} & 1
\end{array}\right], \\
& \mathbf{K}_{c 1}^{i}=k_{p_{i}}\left[\begin{array}{ccc}
1 & 0 & -\sin \varphi_{p_{i}} \\
0 & 1 & \cos \varphi_{p_{i}} \\
-\sin \varphi_{p_{i}} & \cos \varphi_{p_{i}} & 1
\end{array}\right], \\
& \mathbf{K}_{s 1}^{i}
\end{aligned}
$$$$
=k_{s p_{i}}(t)\left[\begin{array}{ccc}
\sin ^{2} \varphi_{s p_{i}} & -\cos \varphi_{s p_{i}} \sin \varphi_{s p_{i}} & -\sin \varphi_{s p_{i}} \\
-\cos \varphi_{s p_{i}} \sin \varphi_{s p_{i}} & \cos ^{2} \varphi_{s p_{i}} & \cos \varphi_{s p_{i}} \\
-\sin \varphi_{s p_{i}} & \cos \varphi_{s p_{i}} & 1
\end{array}\right],
$$$$
\mathbf{K}_{c 2}^{i}=k_{p_{i}}\left[\begin{array}{ccc}
-\cos \varphi_{p_{i}} & \sin \varphi_{p_{i}} & 0 \\
-\sin \varphi_{p_{i}} & -\cos \varphi_{p_{i}} & 0 \\
0 & -1 & 0
\end{array}\right] \text {, }
$$$$
\mathbf{K}_{s 2}^{i}=k_{s p_{i}}(t)\left[\begin{array}{ccc}
\sin \varphi_{s p_{i}} \sin \alpha_{s} & \sin \varphi_{s p_{i}} \cos \alpha_{s} & -\sin \varphi_{s p_{i}} \\
-\cos \varphi_{s p_{i}} \sin \alpha_{s} & -\cos \varphi_{s p_{i}} \cos \alpha_{s} & -\cos \varphi_{s p_{i}} \\
-\sin \alpha_{s} & -\cos \alpha_{s} & 1
\end{array}\right] \text {, }
$$$$
\mathbf{K}_{r 2}^{i}=k_{r p_{i}}(t)\left[\begin{array}{ccc}
-\sin \varphi_{r p_{i}} \sin \alpha_{r} & \sin \varphi_{r p_{i}} \cos \alpha_{r} & \sin \varphi_{r p_{i}} \\
\cos \varphi_{r p_{i}} \sin \alpha_{r} & -\cos \varphi_{r p_{i}} \cos \alpha_{r} & -\cos \varphi_{r p_{i}} \\
\sin \alpha_{r} & \cos \alpha_{r} & -1
\end{array}\right] \text {, }
$$$$
\mathbf{K}^{i}=\mathbf{K}_{c 3}^{i}+\mathbf{K}_{r 3}^{i}+\mathbf{K}_{s 3}^{i}, \quad \mathbf{K}_{c 3}^{i}=\left[\begin{array}{ccc}
k_{p_{i}} & 0 & 0 \\
0 & k_{p_{i}} & 0 \\
0 & 0 & 0
\end{array}\right]
$$$$
\mathbf{K}_{s 3}^{i}=k_{s p_{i}}(t)\left[\begin{array}{ccc}
\sin ^{2} \alpha_{s} & \cos \alpha_{s} \sin \alpha_{s} & -\sin \alpha_{s} \\
\cos \alpha_{s} \sin \alpha_{s} & \cos ^{2} \alpha_{s} & -\cos \alpha_{s} \\
-\sin \alpha_{s} & -\cos \alpha_{s} & 1
\end{array}\right] \text {, }
$$$$
\mathbf{K}_{r 3}^{i}=k_{r p_{i}}(t)\left[\begin{array}{ccc}
\sin ^{2} \alpha_{r} & -\cos \alpha_{r} \sin \alpha_{r} & -\sin \alpha_{r} \\
-\cos \alpha_{r} \sin \alpha_{r} & \cos ^{2} \alpha_{r} & \cos \alpha_{r} \\
-\sin \alpha_{r} & \cos \alpha_{r} & 1
\end{array}\right] \text {, }
$$

where $k_{j p_{i}}(t)(j=s, r)$ is the meshing stiffness between the part $j$ and the $i$ th planet. $k_{p_{i}}$ is the bear supporting stiffness of the $i$ th planet in the transverse and longitudinal direction. $\alpha_{j}$ is the pressure angle of part j. $\varphi_{s p_{i}}=\varphi_{p i}-\alpha_{s}$ and $\varphi_{r p_{i}}=$ $\varphi_{p i}+\alpha_{r}, \varphi_{p i}$ is illustrated in a sun-planet mesh (Figure 2). $i=$ $1,2, \ldots, N, N$ is the number of the planet gears.

$\mathbf{T}(t)$ represents the force matrix and can be expressed by

$$
\mathbf{T}(t)=\left[0,0, \frac{T_{c}}{r_{c}}, 0,0, \frac{T_{r}}{r_{r}}, 0,0, \frac{T_{s}}{r_{s}}, 0, \ldots, 0\right]^{T},
$$

where $T_{j}(j=c, s, r)$ is the external torques applied on part $j$. $r_{j}$ is the base radius of part $j$.

$\mathbf{F}_{W}(t)$ is the impact force caused by faults, the mathematical expression of which will be given in Section 3 .
$\mathbf{F}_{e}(t)$ is the excitation force induced by manufacturing errors and can be written as

$$
\mathbf{F}_{e}(t)=\left[0, \mathbf{F}_{c}, \mathbf{F}_{s}, \mathbf{F}_{1}, \mathbf{F}_{2}, \ldots, \mathbf{F}_{N}\right],
$$

where

$$
\begin{aligned}
& \mathbf{F}_{c}= {\left[\sum_{i=1}^{N} k_{r p_{i}}(t) e_{r i}(t) \sin \left(\varphi_{r p_{i}}\right),\right.} \\
&\left.-\sum_{i=1}^{N} k_{r p_{i}}(t) e_{r i}(t) \cos \left(\varphi_{r p_{i}}\right),-\sum_{i=1}^{N} k_{r p_{i}}(t) e_{r i}(t)\right]^{T}, \\
& \mathbf{F}_{s}=\left[\sum_{i=1}^{N} k_{s p_{i}}(t) e_{s i}(t) \sin \left(\varphi_{s p_{i}}\right),\right. \\
&\left.-\sum_{i=1}^{N} k_{s p_{i}}(t) e_{s i}(t) \cos \left(\varphi_{s p_{i}}\right),-\sum_{i=1}^{N} k_{s p_{i}}(t) e_{s i}(t)\right]^{T}, \\
& \mathbf{F}_{n}=\{\left\{\sum_{i=1}^{N}-k_{r p_{i}}(t) e_{r i}(t) \sin \left(\alpha_{r}\right)+k_{s p_{i}}(t) e_{s i}(t) \sin \left(\alpha_{s}\right),\right. \\
& \sum_{i=1}^{N} k_{r p_{i}}(t) e_{r i}(t) \sin \left(\alpha_{r}\right)-k_{s p_{i}}(t) e_{s i}(t) \cos \left(\alpha_{s}\right) \\
&\left.\sum_{i=1}^{N} k_{r p_{i}}(t) e_{r i}(t)-k_{s p_{i}}(t) e_{s i}(t)\right\} \\
& n=1,2, \ldots, N
\end{aligned}
$$

$e_{j i}(t)(j=s, r)$ is the errors between the part $j$ and the $i$ th planet and can be written as

$$
\begin{aligned}
& e_{s i}(t)=E_{p_{i} s}(t)-E_{s p_{i}}(t)+e_{s p_{i}}(t) \\
& e_{r i}(t)=E_{r p_{i}}(t)-E_{p_{i} r}(t)+e_{r p_{i}}(t),
\end{aligned}
$$

where $E_{j p_{i}}(t)$ and $E_{p_{i} j}(t)(j=s, r)$ are errors associated with rotational frequencies of gears. A unified mathematical formula which is able to express those errors (gear eccentricities, pitch-line run-out errors, tooth spacing, and indexing errors) was extracted by Inalpolat and Kahraman [17],

$$
\begin{aligned}
& E_{j p_{i}}(t)=E_{j} \sin \left[\frac{f_{\text {mesh }}}{z_{j}} t+\varepsilon_{j}-\psi_{j i}\right], \\
& E_{p_{i} j}(t)=E_{p_{i}} \sin \left[\frac{f_{\text {mesh }}}{z_{p}} t+\varepsilon_{p_{i}} \pm \alpha_{j}\right],
\end{aligned}
$$

where $E_{j}$ and $E_{p_{i}}$ are the amplitudes of these errors, $f_{\text {mesh }}$ is mesh frequency, $z_{j}$ and $z_{p}$ are tooth numbers of the component $j$ and planet, $\varepsilon_{j}$ and $\varepsilon_{p_{i}}$ are phase angles, $\psi_{j i}$ is relative position angle, and the arithmetic sign of the pressure angle $\alpha_{j}$ is plus for $j=r$ and minus for $j=s$.

In (11a), $e_{s p_{i}}$ and $e_{r p_{i}}$ are gear transmission errors. Inalpolat and Kahraman [17] believed that those errors were 
amplitude and frequency modulated by $E_{j p_{i}}(t)$ and $E_{p_{i}}(t)$. Therefore, another equation is able to express the gear transmission errors as given by Inalpolat and Kahraman [17],

$$
\begin{aligned}
& e_{s p_{i}}(t)=A_{s p_{i}} e_{s p_{i}} \sin \left[B_{s p_{i}}(t) f_{\text {mesh }} t+z_{s} \varphi_{p_{i}}+\phi_{s p_{i}}\right] \\
& e_{r p_{i}}(t)=A_{r p_{i}} e_{r p_{i}} \sin \left[B_{r p_{i}}(t) f_{\text {mesh }} t+z_{r} \varphi_{p_{i}}+\gamma_{s r}+\phi_{r p_{i}}\right],
\end{aligned}
$$

where

$$
\begin{aligned}
A_{s p_{i}}(t)= & +\beta_{s} \sin \left[\frac{f_{\text {mesh }}}{z_{s}} t+\phi_{\beta s i}\right] \\
& +\beta_{p i} \sin \left[\frac{f_{\text {mesh }}}{z_{p}} t+\phi_{\beta p i}\right] \\
B_{s p_{i}}(t)= & 1+\widehat{\beta}_{s} \sin \left[\frac{f_{\text {mesh }}}{z_{s}} t+\widehat{\phi}_{\hat{\beta} s i}\right] \\
& +\widehat{\beta}_{p i} \sin \left[\frac{f_{\text {mesh }}}{z_{p}} t+\widehat{\phi}_{\hat{\beta} p i}\right] \\
A_{r p_{i}}(t)= & 1+\beta_{r} \sin \left(\frac{f_{\text {mesh }}}{z_{c}} t+\phi_{\beta r i}\right) \\
& +\vartheta_{p i} \sin \left[\frac{f_{\text {mesh }}}{z_{p}} t+\phi_{\vartheta p i}+\pi\right] \\
B_{r p_{i}}(t)= & 1+\widehat{\beta}_{r} \sin \left[\frac{f_{\text {mesh }}}{z_{c}} t+\widehat{\phi}_{\hat{\beta} r i}\right] \\
& +\widehat{\vartheta}_{r i} \sin \left[\frac{f_{\text {mesh }}}{z_{p}} t+\widehat{\phi}_{\hat{\vartheta} p i}+\pi\right],
\end{aligned}
$$

where $\phi_{j p_{i}}(j=s, r)$ is the phase angle of $e_{j p_{j}}(t), \gamma_{s r}$ is the phase angle between $e_{s p_{i}}(t)$ and $e_{r p_{i}}(t), \beta_{s}, \beta_{p i}, \beta_{r}, \vartheta_{p i}$ are amplitude coefficients, $\phi_{\beta s i}, \phi_{\beta p i}, \phi_{\beta r i}, \phi_{9 p i}$ are the phase angles of amplitude modulation, $\widehat{\beta}_{s}, \widehat{\beta}_{p i}, \widehat{\beta}_{r}, \widehat{\vartheta}_{r i}$ are amplitude coefficients, and $\phi_{\beta r i}, \phi_{\vartheta p i}, \widehat{\phi}_{\hat{\beta} r i}, \widehat{\phi}_{\hat{\vartheta}_{p i}}$ are the phase angles of frequency modulation. The research results by Conry and Seireg [21] showed that the peak-to-peak amplitude of $e_{s p_{i}}(t)$ might experience up to $4-6 \%$ variation due to the influence of $E_{s p_{i}}(t)$ and $E_{p_{i} s}(t)$ even with the modest error magnitudes.

\section{Modeling of Tooth Faults}

In this section, the typical tooth fault of tooth breakage is modeled to illustrate the influence of tooth faults on gear mesh. The tooth breakage can be modeled by the reduction of the time-varying mesh stiffness and the generation of additional impact forces $\left(\mathbf{F}_{w}(t)\right)$.

3.1. The Influence of Tooth Breakage on Time-Varying Stiffness. As shown in Figure 3, a tooth is often considered as a cantilever beam with varying section when calculating

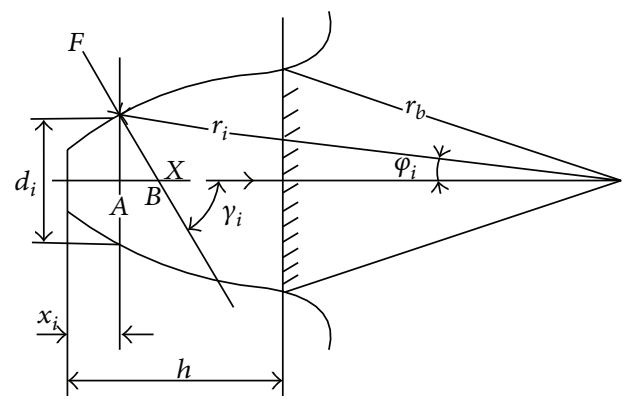

FIgURE 3: The schematic diagram of a cantilever beam.

deformation. Deformation consists of five parts: bending, compressive, shear, fillet-foundation, and contact deformations.

The total deformation of the point $B$ is expressed by

$$
\delta_{B i}=u_{B} \sin \gamma_{i}+v_{B} \cos \gamma_{i}+w_{B} \sin \gamma_{i}+\delta_{f}+\delta_{c},
$$

where $u_{B}, v_{B}, w_{B}, \delta_{f}, \delta_{c}$ represent bending, compressive, shear, fillet-foundation, and contact deformations, respectively, which can be calculated by the method used in [12], $\gamma_{i}$ (see Figure 3) defines the angle between the tooth normal force and the tooth centerline. The stiffness of one gear tooth at point $B$ is calculated as $k_{i}=F / \delta_{B i}$. The total stiffness of one pair of meshing tooth is obtained by $k_{\text {pair }}=\left(k_{1} \times k_{2}\right) /\left(k_{1}+k_{2}\right)$ according to the serial relation. The stiffness when doubleteeth meshing is $k=k_{\text {pair } 1}+k_{\text {pair } 2}$ according to the parallel relation.

The parameters of the studied planetary gear system are listed in Table 1. Young's modulus $E=2.06 \times 10^{11} \mathrm{~Pa}$, Poisson's ratio $v=0.3$. Figure 4 shows the mesh stiffness of the sun gear and ring gear with planet $1\left(k_{s p_{1}}\right.$ and $\left.k_{r p_{1}}\right)$. The planet 1 with and without tooth breakage is considered and the mesh stiffness is seriously affected by tooth breakage.

3.2. The Impact Force Generated by Tooth Breakage. The impact force is generated during the approaching and separation of the meshing teeth [22]. As one gear tooth is broken, the normal gear meshing process is interrupted. When the next gear tooth is going to mesh, additional impacts would occur between the driven and driving gears. The dynamic response of the system will be influenced by the additional impact force. Thus, only the change of mesh stiffness could not reflect the effect of tooth breakage upon the dynamic response of the planetary gear system. In this section, the additional tooth impact forces are modeled.

The additional impact force $\mathbf{F}_{W_{p f}}(t)$ generated by the tooth breakage of the planet gear can be expressed as (planet 1 is assumed to be the fault gear)

$$
\begin{aligned}
\mathbf{F}_{W_{s 1}}(t) & =\left[0,0, \mathbf{F}_{w_{s}}(t), 0, \mathbf{F}_{w_{1}}(t), 0,0\right]^{T} \\
\mathbf{F}_{W_{r 1}}(t) & =\left[0, \mathbf{F}_{w_{r}}(t), 0,0, \mathbf{F}_{w_{1}}(t), 0,0\right]^{T},
\end{aligned}
$$


TABLE 1: Parameters of the planetary gear system.

\begin{tabular}{lcccc}
\hline Parameters & Sun gear & Ring & Carrier & Planets \\
\hline Number of tooth & 17 & 79 & - & 31 \\
Module $(\mathrm{mm})$ & 3 & 3 & - & 3 \\
Face width $(\mathrm{mm})$ & 35 & 35 & - & 35 \\
Mass $(\mathrm{kg})$ & 1.75 & 3.552 & 4.56 & 0.979 \\
Mass moment of inertia $\left(\mathrm{kg} / \mathrm{m}^{2}\right)$ & 1 & $K_{P}=K_{s x}=K_{s y}=K_{r x}=K_{r y}=K_{c x}=K_{c y}=2 \times 10^{8}$ & 0.544 \\
Bearing support stiffness $(\mathrm{N} / \mathrm{m})$ & & & $K_{r u}=10^{9} ; K_{s u}=K_{c u}=2 \times 10^{7}$ & $20^{\circ}$ \\
Torsional stiffness $(\mathrm{N} / \mathrm{m})$ & & $20^{\circ}$ & - & $0^{\circ}$ \\
Pressure angle $\left({ }^{\circ}\right)$ & $20^{\circ}$ & $0^{\circ}$ & - & \\
Helix angle $\left(^{\circ}\right)$ & $0^{\circ}$ & &
\end{tabular}

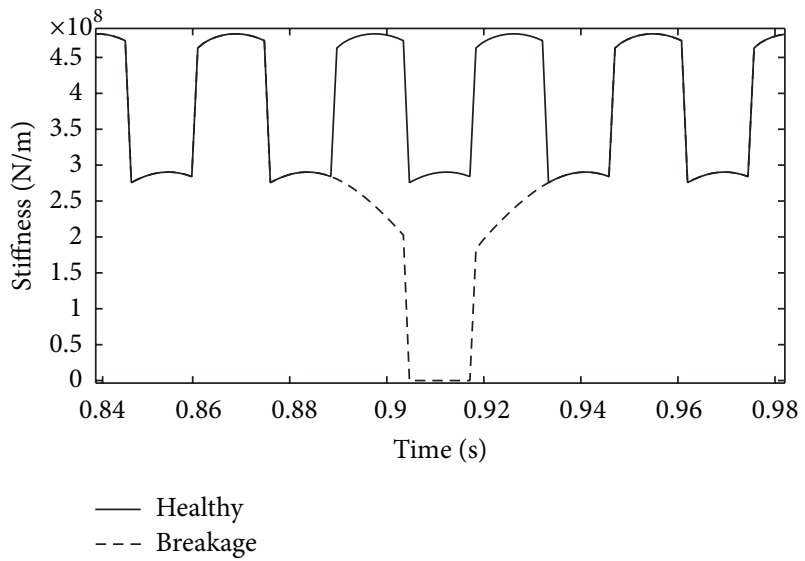

(a)

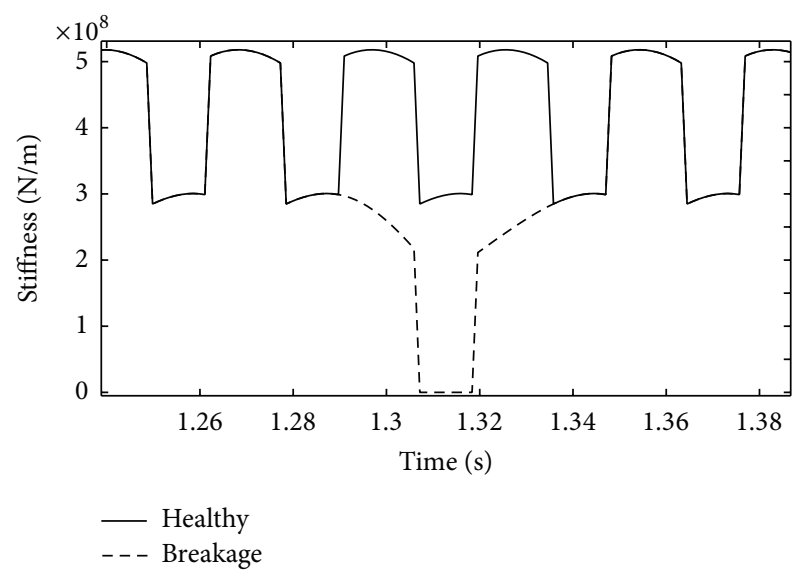

(b)

Figure 4: The mesh stiffness of planet 1 with and without tooth breakage: (a) planet-sun gear pair, (b) planet-ring gear pair.

where

$$
\begin{aligned}
\mathbf{F}_{w_{s}}(t)= & {\left[F_{s 1}(t) \sin \left(\varphi_{s p_{1}}\right),-F_{s 1}(t) \cos \left(\varphi_{s p_{1}}\right),-F_{s 1}(t)\right]^{T} } \\
\mathbf{F}_{w_{r}}(t)= & {\left[F_{r 1}(t) \sin \left(\varphi_{r p_{1}}\right),-F_{r 1}(t) \cos \left(\varphi_{r p_{1}}\right), F_{r 1}(t)\right]^{T} } \\
\mathbf{F}_{w_{1}}(t)= & {\left[-F_{r 1}(t) \sin \left(\alpha_{r}\right)+F_{s 1}(t) \sin \left(\alpha_{s}\right), F_{r 1}(t) \sin \left(\alpha_{r}\right)\right.} \\
& \left.+F_{s 1}(t) \cos \left(\alpha_{s}\right), F_{r 1}(t)-F_{s 1}(t)\right]^{T}
\end{aligned}
$$

where $\mathbf{F}_{W_{j 1}}(t)$ represents the impact force vector of gear $j$ $(j=s, r), F_{j 1}(t)(j=s, r)$ denotes the impact force and can be expressed as

$$
F_{j 1}(t)= \begin{cases}C_{j 1}(t) & t_{I b_{j 1}}<t<t_{I f_{j 1}} \\ 0 & \text { else, }\end{cases}
$$

where $t_{I b_{j 1}}$ and $t_{I f_{j 1}}$ represent the beginning and finishing time of the impact force of gear $j$. The relationship between $C_{r}(t)$ and $C_{s}(t)$ is expressed by $C_{r}(t)=H(t) C_{s}(t)$. Because the impact acceleration will be generated, $H(t)>1$ when the fault tooth meshes with ring gear and $H(t)<1$ when the fault tooth meshes with sun gear.

The additional impact force $\mathbf{F}_{W_{r f}}(t)$ generated by the tooth breakage of the ring gear could be expressed by

$$
\begin{aligned}
& \mathbf{F}_{W_{r 1}}(t)=\left[0, \mathbf{F}_{w_{r 1}}(t), \mathbf{F}_{w_{s 1}}(t), 0, \mathbf{F}_{w_{1}}(t), 0,0\right]^{T} \\
& \mathbf{F}_{W_{r 2}}(t)=\left[0, \mathbf{F}_{w_{r 2}}(t), \mathbf{F}_{w_{s 2}}(t), 0,0, \mathbf{F}_{w_{2}}(t), 0\right]^{T} \\
& \mathbf{F}_{W_{r 3}}(t)=\left[0, \mathbf{F}_{w_{r 3}}(t), \mathbf{F}_{w_{s 3}}(t), 0,0,0, \mathbf{F}_{w_{3}}(t)\right]^{T},
\end{aligned}
$$

where

$$
\begin{gathered}
\mathbf{F}_{w_{r i}}(t)=\left[F_{r i}(t) \sin \left(\varphi_{r p_{i}}\right),-F_{r i}(t) \cos \left(\varphi_{r p_{i}}\right),-F_{r i}(t)\right]^{T} \\
\mathbf{F}_{w_{s i}}(t)=\left[F_{s i}(t) \sin \left(\varphi_{s p_{i}}\right),-F_{s i}(t) \cos \left(\varphi_{s p_{i}}\right),-F_{s i}(t)\right]^{T} \\
\mathbf{F}_{w_{i}}(t)=\left[-F_{r i}(t) \sin \left(\alpha_{r}\right)+F_{s i}(t) \sin \left(\alpha_{s}\right),\right. \\
\left.F_{r i}(t) \sin \left(\alpha_{r}\right)+F_{s i}(t) \cos \left(\alpha_{s}\right), F_{r i}(t)-F_{s i}(t)\right]^{T},
\end{gathered}
$$

where $\mathbf{F}_{W r i}(t)$ represents the impact force matrix when planet $i$ meshes with ring gear, $F_{s i}(t)$ and $F_{r i}(t)$ are the pulsating impact forces, the mathematical expressions of which are similar to (15) and could be expressed by

$$
F_{j i}(t)= \begin{cases}C_{j i}(t) & t_{I b_{j i}}<t<t_{I f_{j i}} \\ 0 & \text { else. }\end{cases}
$$

Similarly, there are several coefficients $H_{j i}(t)$ used to describe the relation among $C_{j i}(t)$. The starting time of $F_{s i}(t)$ and $F_{r i}(t)$ varies with $i$. 

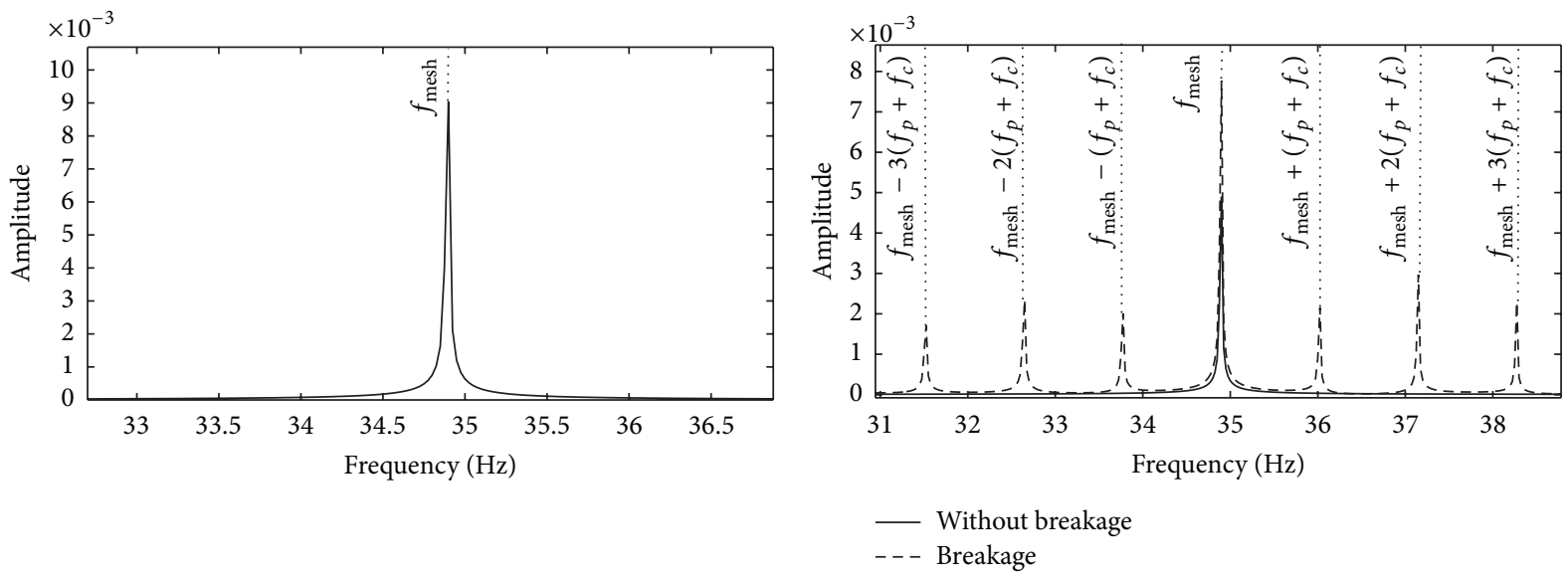

(a)

(b)

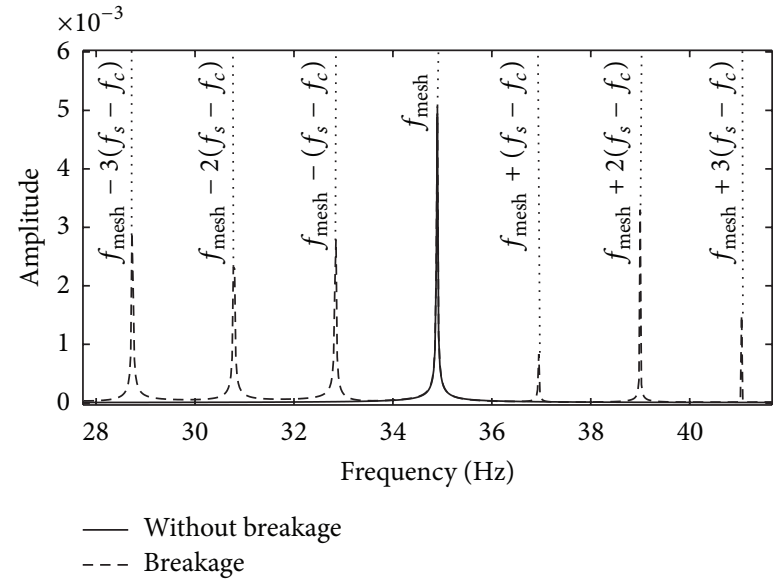

(c)

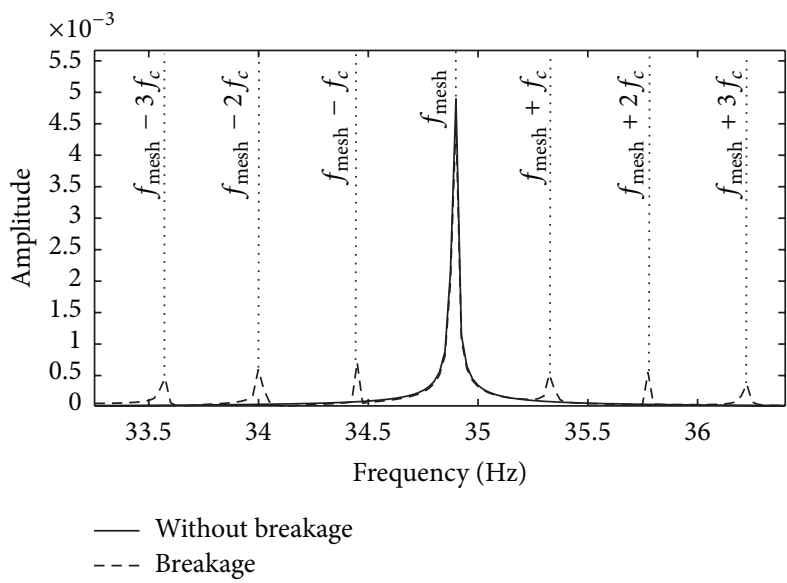

(d)

FIGURE 5: The acceleration spectrum of the ring gear in longitudinal direction in the system with and without tooth breakages (without errors): (a) without breakage, (b) planet gear with and without tooth breakages, (c) sun gear with and without tooth breakages, (d) ring gear with and without tooth breakages.

The impact force generated by the tooth breakage of the sun gear is $\mathbf{F}_{W_{s f}}(t)$, the mathematical expression of which is the same as that of $\mathbf{F}_{W_{r f}}(t)$. However, the values for beginning and finishing times of $F_{j i}(t)$ are different.

The research results by Yao and Wei [23] showed that the action time of impact force is usually about $5-10 \%$ of one mesh cycle $\left(T_{m}\right)$. Wang et al. [24] pointed out that the maximal impact force induced by tooth breakage is about twice the average mesh force. Thus, we can assume $t_{I f_{j i}}-t_{I b_{j i}}=$ $T_{m} / 10$ and $C_{j i}(t)=2 \bar{F}_{j p_{i}}=$ const.

\section{The Dynamic Response of Planetary Gear System without Errors}

Physical parameters of the studied planetary gear system are listed in Table 1. The ring gear is fixed and there are three planets rotating around the sun gear. The speed of the input part (carrier) is $2.77 \mathrm{rad} / \mathrm{s}\left(\Omega_{c}\right)$ and the load of the output part (sun gear) is $200 \mathrm{~N} \cdot \mathrm{m}$. The rotating frequency of the carrier $\left(f_{c}\right)$, sun gear $\left(f_{s}\right)$, planets $\left(f_{p}\right)$, and the mesh frequency $\left(f_{\text {mesh }}\right)$ can be calculated by

$$
\begin{gathered}
f_{c}=\frac{\Omega_{c}}{2 \pi}, \\
f_{s}=\frac{z_{s}+z_{r}}{z_{s}} f_{c}, \\
f_{p}=\frac{f_{s} z_{s}-f_{c}\left(z_{s}+z_{p}\right)}{z_{p}}, \\
f_{\text {mesh }}=f_{c} z_{r} .
\end{gathered}
$$

Obviously, these frequency values are $f_{c}=0.441 \mathrm{~Hz}, f_{s}=$ $2.49 \mathrm{~Hz}$, and $f_{p}=0.68 \mathrm{~Hz}$, respectively, and the mesh frequency is $f_{\text {mesh }}=34.9 \mathrm{~Hz}$.

The errors $e_{s p_{i}}, e_{r p_{i}}, E_{p_{i}}, E_{s}, E_{r}$ are set to be zero. The dynamic equation of the model is solved by the NEWMARK method. Figure 5(a) shows the acceleration spectrum of the ring gear in longitudinal direction (without breakage). The spectrum is occupied by the mesh frequency and no 


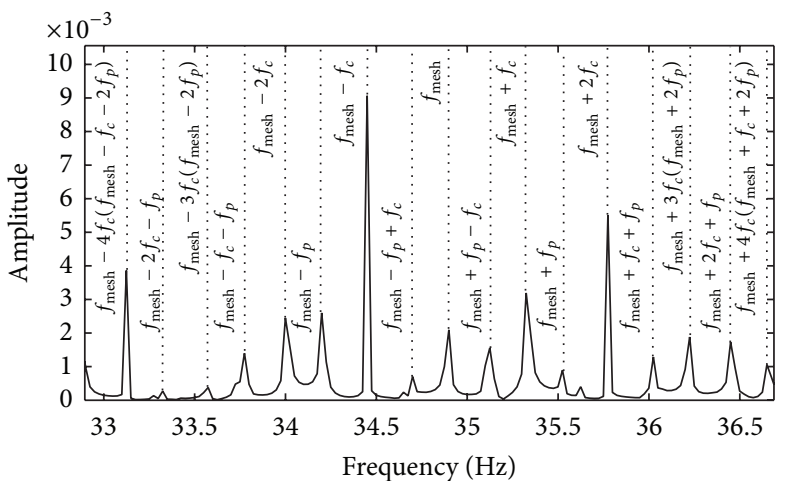

(a)

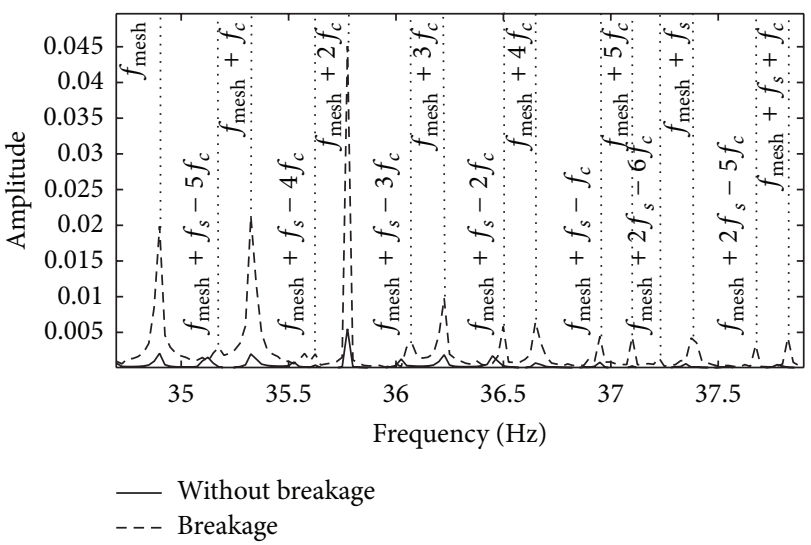

(c)

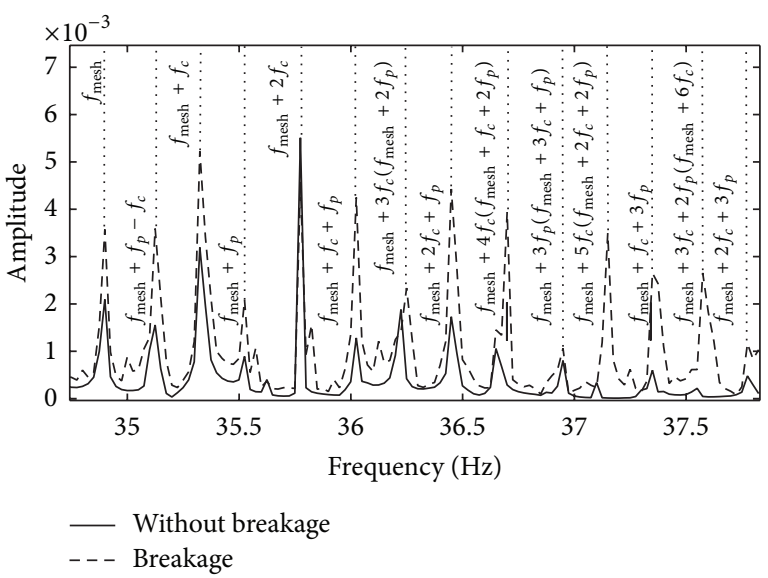

(b)

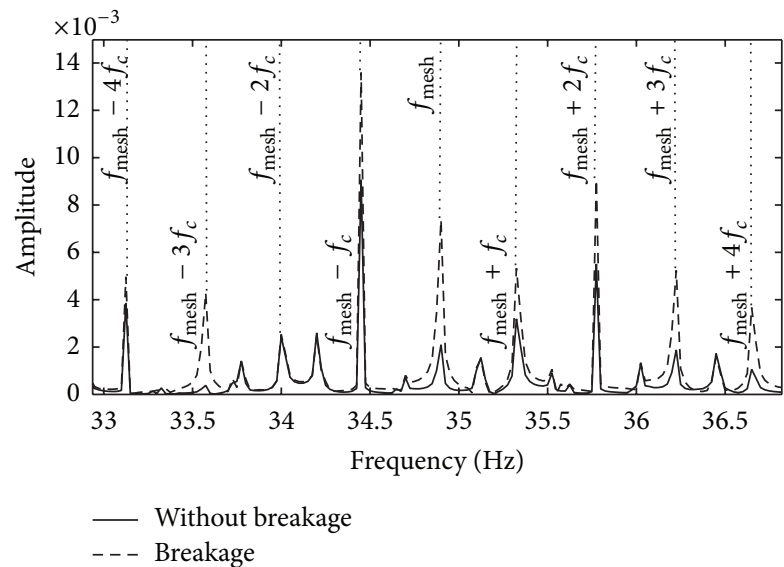

(d)

FIGURE 6: The spectrum of the system with and without tooth breakages (with errors): (a) without breakage, (b) planet gear with and without tooth breakages (the right side of $f_{\text {mesh }}$ ), (c) sun gear with and without tooth breakages (the right side of $f_{\text {mesh }}$ ), (d) ring gear with and without tooth breakages.

TABLE 2: Parameters of the parallel-stage gear.

\begin{tabular}{lcccc}
\hline Level & \multicolumn{2}{c}{ Stage1 } & \multicolumn{2}{c}{ Stage2 } \\
Gear & Input & Output & Input & Output \\
\hline Number of tooth & 73 & 21 & 66 & 23 \\
\hline
\end{tabular}

sidebands are found around the mesh frequency. Then tooth breakage is added to the planet gear, sun gear, and ring gear successively, and the acceleration spectrum (the ring gear in longitudinal direction) is showed in Figures 5(b)-5(d). Compared with the spectrum of the normal system (without breakage), modulation sidebands appeared in the spectrum of the breakage system and the peaks of the sidebands are at the frequencies of $f_{\text {mesh }} \pm n\left(f_{p}+f_{c}\right), f_{\text {mesh }} \pm n\left(f_{s}-f_{c}\right)$ and $f_{\text {mesh }} \pm n f_{c}(n=1,2, \ldots)$ successively. Therefore, the mesh frequency $f_{\text {mesh }}$ is modulated by those frequencies, which are caused by the influence of tooth breakage on mesh stiffness and additional impact force. So, the sidebands of the planetary gear system are comparatively simple if the errors are not included in the system.

\section{The Dynamic Response of Planetary Gear System with Errors}

In order to display the modulation effect of errors more reasonably, a formula was given by Inalpolat and Kahraman [17] to express the acceleration response of the system

$$
a(t)=\sum_{i=1}^{N} w_{i}(t)\left(\bar{C}_{i} k_{s p_{i}}(t) \delta_{s p_{i}}(t)+\bar{D}_{i} k_{r p_{i}}(t) \delta_{r p_{i}}(t)\right)
$$

where $\bar{C}_{i}$ and $\bar{D}_{i}$ are constants, $\delta_{s p_{i}}(t)$ and $\delta_{r p_{i}}(t)$ are defined as

$\delta_{s p_{i}}(t)=y_{s} \cos \varphi_{s p_{i}}-x_{s} \sin \varphi_{s p_{i}}-x_{i} \sin \alpha_{s}+u_{s}+u_{i}-e_{s i}(t)$,

$\delta_{r p_{i}}(t)=y_{r} \cos \varphi_{r p_{i}}-x_{r} \sin \varphi_{r p_{i}}-x_{i} \sin \alpha_{r}+u_{r}-u_{i}+e_{r i}(t)$

$x_{j}, y_{j}, u_{j}(j=s, r, i)$ can be obtained by the dynamic model. 


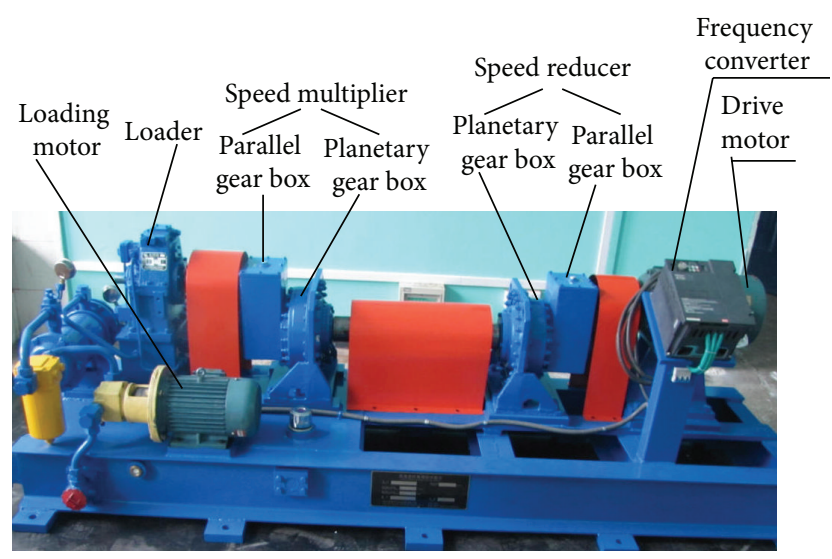

FIGURE 7: The test bench of the planetary gear system.

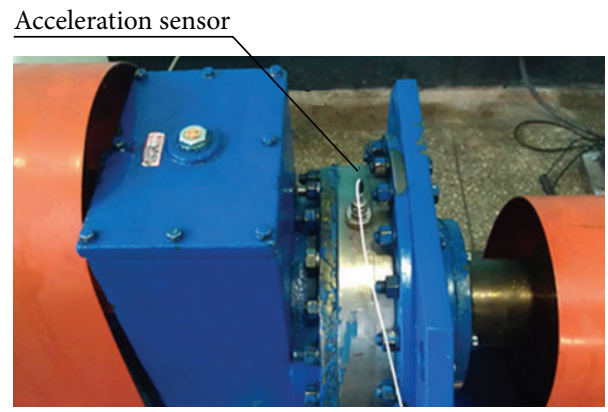

(a)

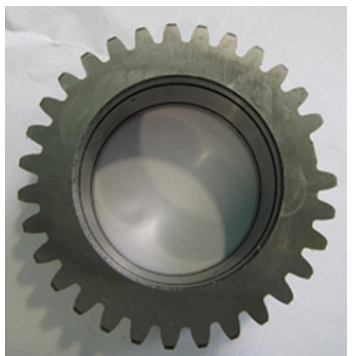

(b)

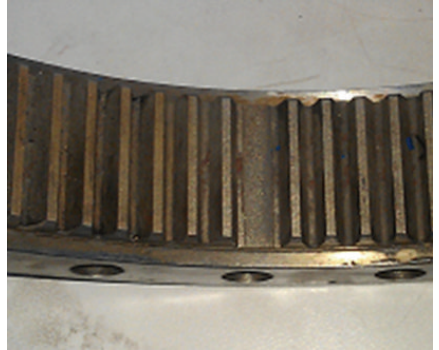

(c)
FIgURE 8: The acceleration sensor and breakage tooth: (a) acceleration sensor position, (b) planet gear with tooth breakage, and (c) ring gear with tooth breakage.

$w_{i}(t)$ is a weighting function for a planet gear positioned at the angle of $\varphi_{p_{i}}$ and $w_{i}(t)$ can be defined as

$$
w_{i}(t)=W\left(t-\frac{\varphi_{p_{i}}}{f_{c}}\right) U_{i}(t),
$$

where $W(t)$ is a Hanning function used to represent the influence of rolling planets on fixed accelerometer (scrolling

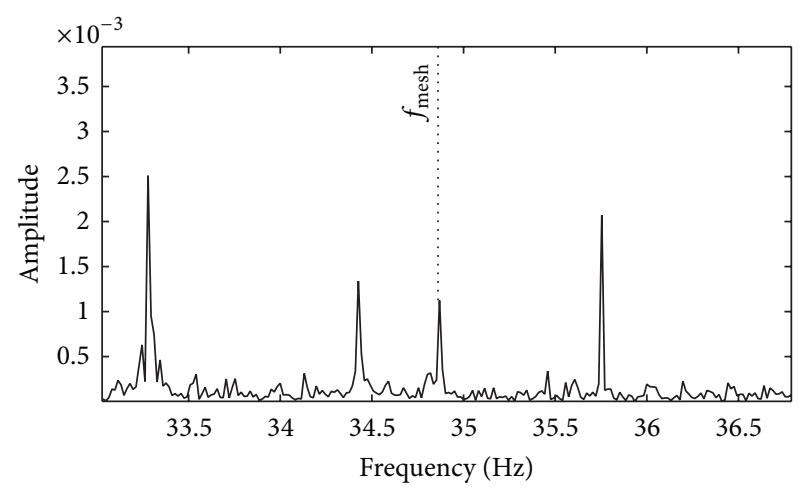

FIGURE 9: The acceleration spectrum of the normal system.

effect) and $U_{i}(t)$ is the unit step function. Both of them are given as follows:

$$
\begin{gathered}
W(t)=\frac{1}{2}-\frac{1}{2}\left[\cos \left(2 \pi N f_{c} t\right)\right] \\
U_{i}(t)=\sum_{n=1}^{\infty}\left\{u\left[t-\left(\frac{(n-1) N+i-1}{N}\right) T_{c}\right]\right. \\
\left.-u\left[t-\left(\frac{(n-1) N+i}{N}\right) T_{c}\right]\right\},
\end{gathered}
$$

where $u(t-a)=1$ for $t>a$ and $u(t-a)=0$ for $t<a, N$ is the number of planets. In this study, $N$ is equal to 3 and $i$ is 1 , 2 , and 3 .

Run-out errors of the planets, sun gear, and ring gear are taken into account and $e_{s p_{i}}=0.6 \mu \mathrm{m}, e_{r p_{i}}=0.3 \mu \mathrm{m}$, $E_{p_{i}}=10 \mu \mathrm{m}$, and $E_{s}=E_{r}=15 \mu \mathrm{m}$. According to Conry's study [21], modulation coefficients are set as $\beta_{s}=0.033$, $\phi_{\beta s i}=2(i-1) \pi / 3, \beta_{p i}=0.034, \phi_{\beta p i}=2(i-2) \pi / 3, \vartheta_{p i}=0.033$, $\phi_{9 p i}=2(i-2) \pi / 3, \beta_{r}=0.035, \phi_{\beta r i}=2(i-1) \pi / 3$ and all other modulation coefficients are set to be zero. The dynamic equation of the model is solved to get the $\delta_{s p_{i}}(t)$ and $\delta_{r p_{i}}(t)$, then acceleration is calculated by (20). Figure 6(a) shows the system acceleration spectrum of a normal system (without breakage). Compared with Figure 5(a), complex sidebands appeared around the mesh frequency due to the existence of errors. They are symmetrically arranged on both sides of the mesh frequency. The sidebands of the system have undergone tremendous changes when errors are taken into consideration. The phenomenon is bound to influence the fault characteristic frequencies of the system components.

Figure 6(b) shows the acceleration spectrum of the system with and without tooth breakage on planet 1 . Since the mesh frequency $f_{\text {mesh }}$ is modulated by the frequencies of weighting function (which characteristic frequencies are $m f_{c}$ ) and the frequencies of $n\left(f_{p}+f_{c}\right)$ (caused by the influence of tooth breakage on mesh stiffness and additional impact force), the amplitudes of the tooth breakage system are much larger than those of the normal (without breakage) system at the frequencies of $f_{\text {mesh }} \pm m f_{c} \pm n\left(f_{p}+f_{c}\right)(m, n=1,2, \ldots)$. Therefore, the fault characteristic frequencies of the planet gear are $f_{p f}=f_{\text {mesh }} \pm m f_{c} \pm n\left(f_{p}+f_{c}\right)$ and can be simplified as $f_{\text {mesh }} \pm m f_{c} \pm n f_{p}(m, n=1,2, \ldots)$. 


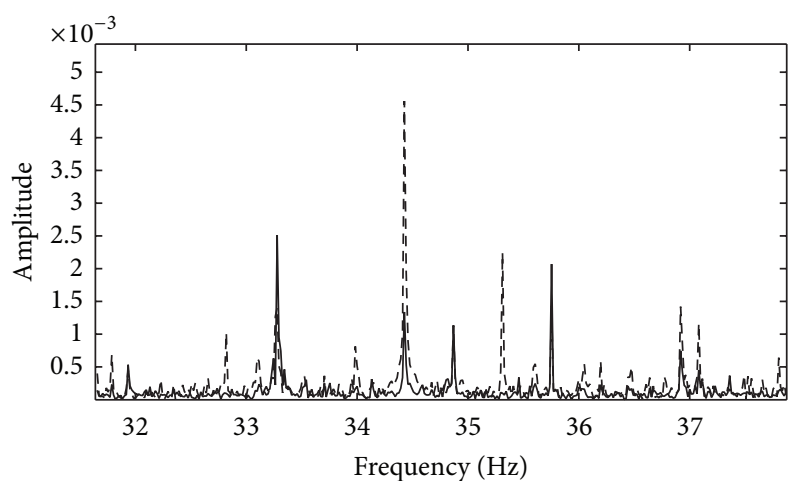

- Without breakage
-- Breakage

(a)

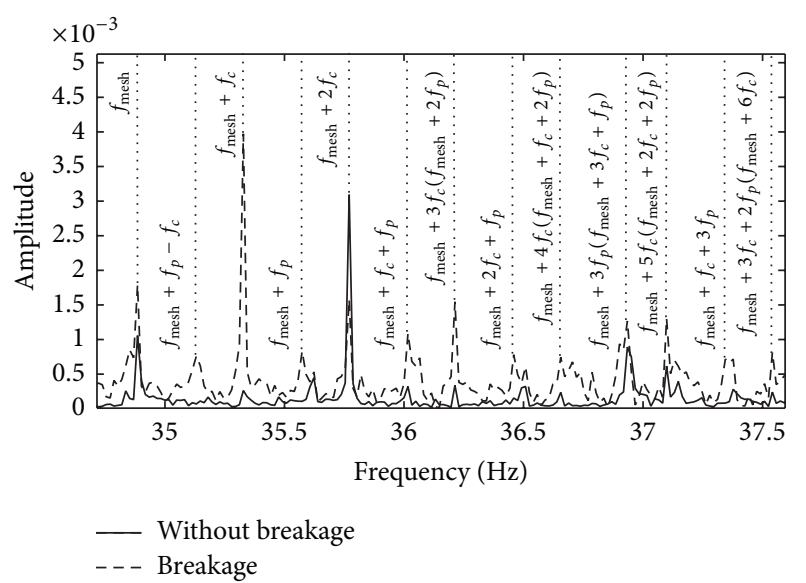

(c)

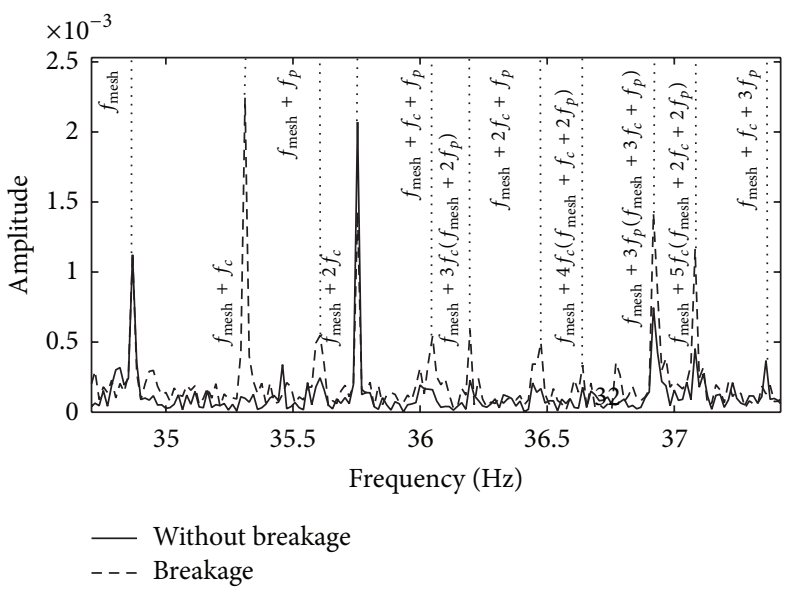

(b)

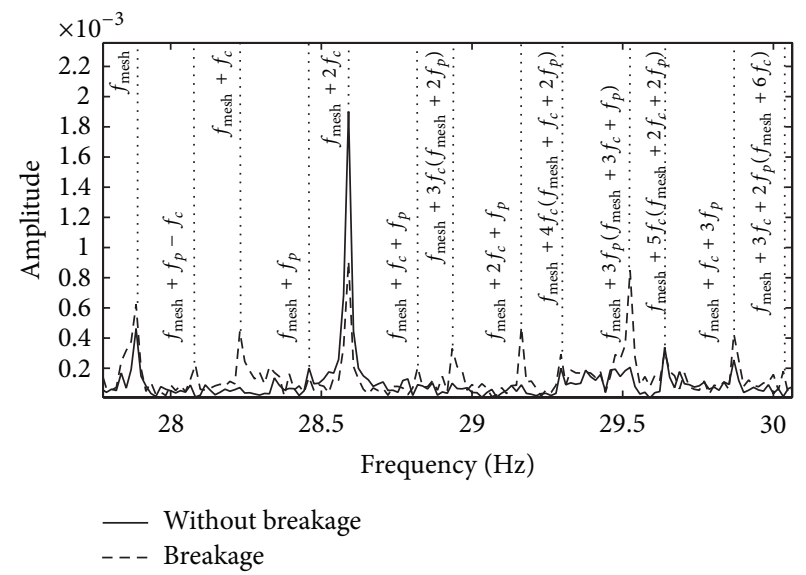

(d)

Figure 10: The spectrum of the system with and without tooth breakage on planet at different speeds and loads: (a) load $=200 \mathrm{~N} \cdot \mathrm{m}$, input speed $=2.74 \mathrm{rad} / \mathrm{s}$, (b) zoom the right side of (a), (c) load $=229 \mathrm{~N} \cdot \mathrm{m}$, input speed $=2.74 \mathrm{rad} / \mathrm{s}$, and $(\mathrm{d})$ load $=229 \mathrm{~N} \cdot \mathrm{m}, \mathrm{input}$ speed $=2.22 \mathrm{rad} / \mathrm{s}$.

Likewise, the amplitudes of the sun gear tooth breakage system are found to be larger than those of the normal system (without breakage) at the frequencies of $f_{\text {mesh }} \pm n f_{c} \pm m f_{s}$ $(m, n=1,2, \ldots)$ in Figure 6(c). The main reason is that the mesh frequency $f_{\text {mesh }}$ is modulated by the frequencies of weighting function $\left(m f_{c}\right)$ and the frequencies of $n\left(f_{s}-\right.$ $f_{c}$ ) (caused by the influence of tooth breakage on mesh stiffness and additional impact force). Therefore, the fault characteristic frequencies of the sun gear are $f_{\text {mesh }} \pm m f_{c} \pm$ $n\left(f_{s}-f_{c}\right)$ and can be as $f_{s f}=f_{\text {mesh }} \pm n f_{c} \pm m f_{s}(m, n=$ $1,2, \ldots)$.

Similarly, because the mesh frequency $f_{\text {mesh }}$ is modulated by the frequencies of the weighting function $m f_{c}$ and the frequencies of $n f_{c}$ (caused by the influence of tooth breakage on mesh stiffness and additional impact force), the amplitudes of the ring gear tooth breakage system are larger than those of the normal system (without breakage) at frequencies of $f_{\text {mesh }} \pm n f_{c} \pm m f_{c}(m, n=1,2, \ldots)$, as shown in Figure 6(d). Therefore, the fault characteristic frequencies of the ring gear are $f_{r f}=f_{\text {mesh }} \pm n f_{c} \pm m f_{c}$ and can be simplified as $f_{r f}=$ $f_{\text {mesh }} \pm n f_{c}(n=1,2, \ldots)$.

\section{Experimental Validations}

Figure 7 shows the test bench of a planetary gear system. The drive motor is controlled by frequency converter and provides power for the system. The speed of the system is slowed down by the speed reducer and speeded up by the speed multiplier. A loading motor continuously pumps high-pressure oil into the loader to induce a workload. The structure and the size of the speed multiplier are proportional to the gearbox of a real wind turbine and it consists of a planetary and a parallel gear boxes. The parallel gear box is composed of two pairs of parallel-stage gearing. The parameters of the planetary gear system are listed in Table 1 and those of the parallel-stage are listed in Table 2. The structure style and parameter setting of the speed reducer are the same as those of the speed multiplier.

As shown in Figure 8(a), an acceleration sensor is mounted on the planetary gear box of the speed multiplier. The tooth breakage of planet gear and ring gear are added to the planetary gear system separately, as shown in Figures 8(b) and $8(\mathrm{c})$. Vibration signals are collected for a normal mode 


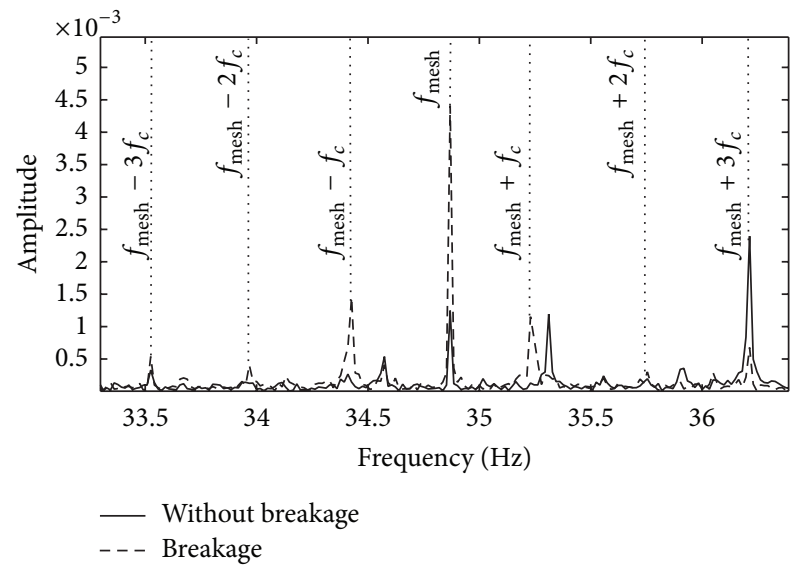

(a)

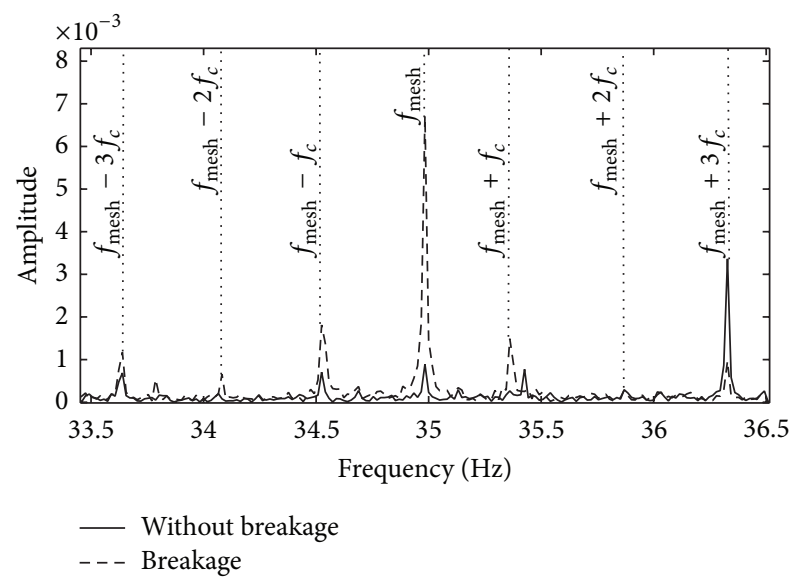

(b)

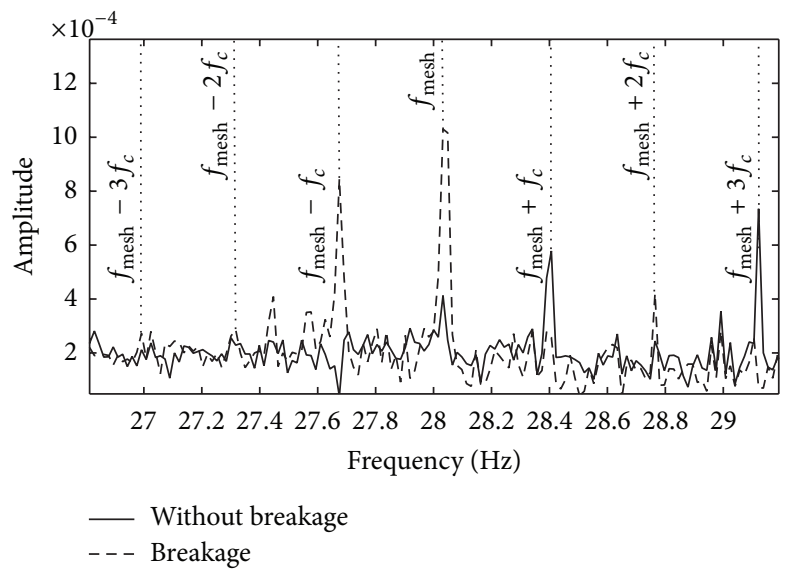

(c)

FIGURE 11: The spectrum of the system with and without tooth breakage on ring gear at different loads and speeds: (a) load $=200 \mathrm{~N} \cdot \mathrm{m}$, input speed $=2.77 \mathrm{rad} / \mathrm{s}$, (b) load $=229 \mathrm{~N} \cdot \mathrm{m}$, input speed $=2.77 \mathrm{rad} / \mathrm{s}$, and $(\mathrm{c})$ load $=229 \mathrm{~N} \cdot \mathrm{m}$, input speed $=2.24 \mathrm{rad} / \mathrm{s}$.

(without breakage) and the two fault modes. The sampling frequency is $16384 \mathrm{~Hz}$.

Figure 9 shows the acceleration spectrum of the normal system (without breakage). The drive motor speed is $156.24 \mathrm{rad} / \mathrm{s}$ and the input speed of the speed multiplier is $2.77 \mathrm{rad} / \mathrm{s}$. Therefore, the mesh frequency is $34.9 \mathrm{~Hz}$. Figure 9 shows that sidebands appeared around the mesh frequency. The results indicate the existence of errors in the system.

6.1. Planet Gear with Tooth Breakage. Figure 10 shows the acceleration spectrum of the system with and without tooth breakage on planet.

In Figure 10(a), the load and the speed of the system are $200 \mathrm{~N} \cdot \mathrm{m}$ and $2.74 \mathrm{rad} / \mathrm{s}$, respectively. Therefore, the mesh frequency is $34.87 \mathrm{~Hz}$. Compared with the amplitudes of the normal system (without breakage), those of the tooth breakage system change significantly. In order to make it more clear, the right side of Figure 10(a) is zoomed in, as shown in Figure 10(b). All amplitudes increase at the frequencies of $f_{\text {mesh }} \pm m f_{c} \pm n f_{p}(m, n=1,2, \ldots)$, except for the frequencies of $f_{\text {mesh }}+2 f_{c}$ and $f_{\text {mesh }}+f_{c}+3 f_{p}$.
Then, the load is increased to $229 \mathrm{~N} \cdot \mathrm{m}$ and the speed remains the same. The differences of amplitudes between the normal system and the tooth breakage system are increased at the frequencies of $f_{\text {mesh }} \pm m f_{c} \pm n f_{p}(m, n=1,2, \ldots)$, as shown in Figure 10(c).

Finally, the load is kept unchanged at a value of $229 \mathrm{~N} \cdot \mathrm{m}$ and the input speed is decreased to $2.22 \mathrm{rad} / \mathrm{s}$. Therefore, the mesh frequency is changed to $27.86 \mathrm{~Hz}$. The amplitudes of the tooth breakage system are also larger than those of the normal system at the frequencies of $f_{\text {mesh }} \pm m f_{c} \pm n f_{p}(m, n=1,2, \ldots)$ significantly, as shown in Figure 10(d).

Figure 10 verified the correctness of the simulation results in Section 5. The fault characteristic frequencies of the planet gear with tooth breakage are $f_{p f}=f_{\text {mesh }} \pm m f_{c} \pm n f_{p}(m, n=$ $1,2, \ldots)$.

6.2. Ring Gear with Breakage. Figure 11 shows the acceleration spectrum of the system with and without tooth breakage on the ring gear.

In Figure 11, the load and the speed of the system are $200 \mathrm{~N} \cdot \mathrm{m}$ and $2.77 \mathrm{rad} / \mathrm{s}$, respectively; therefore, the mesh 
frequency is $34.9 \mathrm{~Hz}$, similar with the method used in Section 6.1. The amplitudes of the acceleration spectrum are compared between the normal system and the tooth breakage system. The fault characteristic frequencies of $f_{\text {mesh }} \pm n f_{c}$ $(n=1,2, \ldots)$ are further confirmed by the experiment results.

The change law of amplitudes with loads and speeds of the normal and the tooth breakage systems is same as the law described in Section 6.1. The conclusion can be obtained through Figures 11(a)-11(c).

\section{Conclusions}

Detection and localization of tooth fault on wind turbine planetary gear system are investigated numerically and experimentally in this paper. Through detailed comparisons on both numerical and experimental results, the fault characteristic frequencies can be obtained after considering the gear manufacturing errors.

(1) The sidebands of the planetary gear system become much more complex when manufacturing errors are taken into consideration.

(2) The fault characteristic frequencies of planet gears, sun gear and ring gear with tooth breakage are $f_{p f}=$ $f_{\text {mesh }} \pm m f_{c} \pm n f_{p}, f_{s f}=f_{\text {mesh }} \pm n f_{c} \pm m f_{s}$ and $f_{r f}=f_{\text {mesh }} \pm n f_{c}$, respectively.

(3) As the fault characteristic frequencies for the sun, planet, and ring gears are different, so the tooth breakage fault could also be located by monitoring the values of these frequencies.

The above results are useful for the detection and localization of tooth faults in actual planetary gear system of wind turbines.

\section{Conflict of Interests}

The authors declare that there is no conflict of interests regarding the publication of this paper.

\section{Acknowledgments}

The research work described in this paper was supported by Natural Science Foundation of China under Grant 51335006, Tsinghua University Initiative Scientific Research Program under Grant 2011Z08137, and the State Key Laboratory of Tribology Initiative Research Program under Grant SKLT11A02.

\section{References}

[1] J. McNames, "Fourier series analysis of epicyclic gearbox vibration," Journal of Vibration and Acoustics, vol. 124, no. 1, pp. 150152, 2002.

[2] A. Kusiak and W. Li, "The prediction and diagnosis of wind turbine faults," Renewable Energy, vol. 36, no. 1, pp. 16-23, 2011.

[3] C. Hatch, "Improved wind turbine condition monitoring using acceleration enveloping," Orbit, pp. 58-61, 2004.
[4] C. Walford, "Wind turbine reliability: Understanding and minimizing wind turbine operation and maintenance costs," Report SAND2006-1100, Sandia National Laboratories, March 2006.

[5] Y. Lei, D. Kong, J. Lin, and M. J. Zuo, "Fault detection of planetary gearboxes using new diagnostic parameters," Measurement Science and Technology, vol. 23, no. 5, Article ID 055605, 2012.

[6] J. Lin and M. J. Zuo, "Gearbox fault diagnosis using adaptive wavelet filter," Mechanical Systems and Signal Processing, vol. 17, no. 6, pp. 1259-1269, 2003.

[7] W. Y. Liu, W. H. Zhang, J. G. Han, and G. F. Wang, "A new wind turbine fault diagnosis method based on the local mean decomposition," Renewable Energy, vol. 48, pp. 411-415, 2012.

[8] P. D. McFadden and J. D. Smith, "An explanation for the asymmetry of the modulation sidebands about the tooth meshing frequency in epicyclic gear vibration," Proceedings of the Institution of Mechanical Engineers C: Mechanical engineering science, vol. 199, no. 1, pp. 65-70, 1985.

[9] P. D. McFadden, "A technique for calculating the time domain averages of the vibration of the individual planet gears and the sun gear in an epicyclic gearbox," Journal of Sound and Vibration, vol. 144, no. 1, pp. 163-172, 1991.

[10] Z. Feng and M. J. Zuo, "Vibration signal models for fault diagnosis of planetary gearboxes," Journal of Sound and Vibration, vol. 331, no. 22, pp. 4919-4939, 2012.

[11] F. Chaari, T. Fakhfakh, and M. Haddar, "Dynamic analysis of a planetary gear failure caused by tooth pitting and cracking," Journal of Failure Analysis and Prevention, vol. 6, no. 2, pp. 7378, 2006.

[12] F. Chaari, T. Fakhfakh, and M. Haddar, "Analytical investigation on the effect of gear teeth faults on the dynamic response of a planetary gear set," Noise and Vibration Worldwide, vol. 37, no. 8, pp. 9-15, 2006.

[13] Z. Cheng, N. Hu, F. Gu, and G. Qin, "Pitting damage levels estimation for planetary gear sets based on model simulation and grey relational analysis," Transactions of the Canadian Society for Mechanical Engineering, vol. 35, no. 3, pp. 403-417, 2011.

[14] Z. Cheng, N. Q. Hu, M. J. Zuo, and B. Fan, "Crack level estimation approach for planetary gear sets based on simulation signal and GRA," Journal of Physics: Conference Series, vol. 364, Article ID 012076, 2012.

[15] S. Jia and I. Howard, "Comparison of localised spalling and crack damage from dynamic modelling of spur gear vibrations," Mechanical Systems and Signal Processing, vol. 20, no. 2, pp. 332349, 2006.

[16] H. Ligata, A. Kahraman, and A. Singh, "An experimental study of the influence of manufacturing errors on the planetary gear stresses and planet load sharing," Journal of Mechanical Design, Transactions of the ASME, vol. 130, no. 4, Article ID 041701, 2008.

[17] M. Inalpolat and A. Kahraman, "A dynamic model to predict modulation sidebands of a planetary gear set having manufacturing errors," Journal of Sound and Vibration, vol. 329, no. 4, pp. 371-393, 2010.

[18] F. Chaari, T. Fakhfakh, R. Hbaieb, J. Louati, and M. Haddar, "Influence of manufacturing errors on the dynamic behavior of planetary gears," International Journal of Advanced Manufacturing Technology, vol. 27, no. 7-8, pp. 738-746, 2006.

[19] J. Lin and R. G. Parker, "Analytical characterization of the unique properties of planetary gear free vibration," Journal of Vibration and Acoustics, vol. 121, no. 3, pp. 316-321, 1999. 
[20] T. Barszcz and R. B. Randall, "Application of spectral kurtosis for detection of a tooth crack in the planetary gear of a wind turbine," Mechanical Systems and Signal Processing, vol. 23, no. 4, pp. 1352-1365, 2009.

[21] T. F. Conry and A. Seireg, "A mathematical programming technique for the evaluation of load distribution and optimal modifications for gear systems," Journal of Manufacturing Science and Engineering, vol. 95, no. 4, pp. 1115-1122, 1973.

[22] T. J. Lin, H. Ou, and R. F. Li, "A finite element method for 3D static and dynamic contact/impact analysis of gear drives," Computer Methods in Applied Mechanics and Engineering, vol. 196, no. 9-12, pp. 1716-1728, 2007.

[23] W. X. Yao and R. Z. Wei, "Research on the mesh impact of spur gear," Journal of Vibration and Shock, vol. 4, pp. 57-61, 1990 (Chinese).

[24] L. X. Wang, L. L. Jun, and S. Z. Le, "Impact incentive research of broken teeth gear," Coal Mine Machinery, vol. 334, pp. 264-266, 2012 (Chinese). 

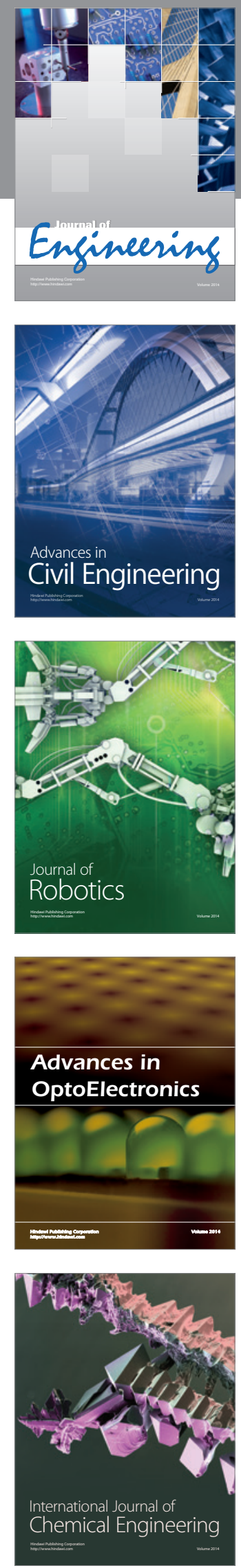

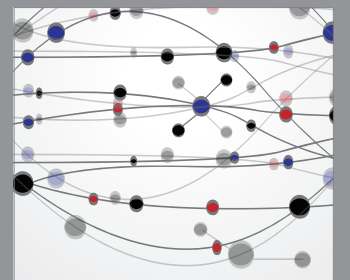

The Scientific World Journal
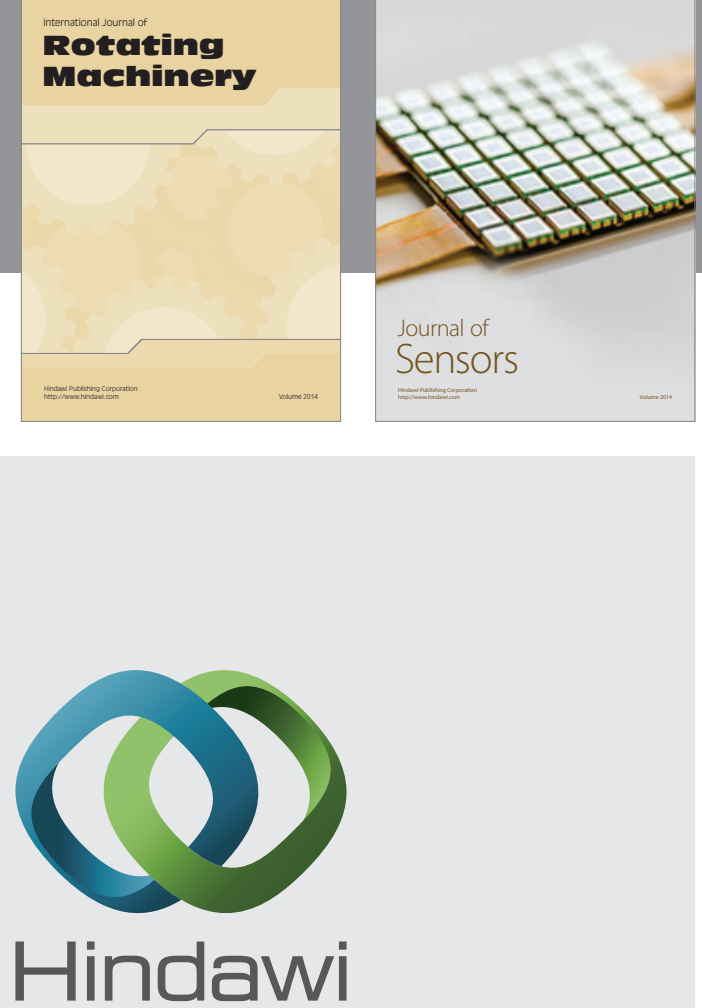

Submit your manuscripts at http://www.hindawi.com
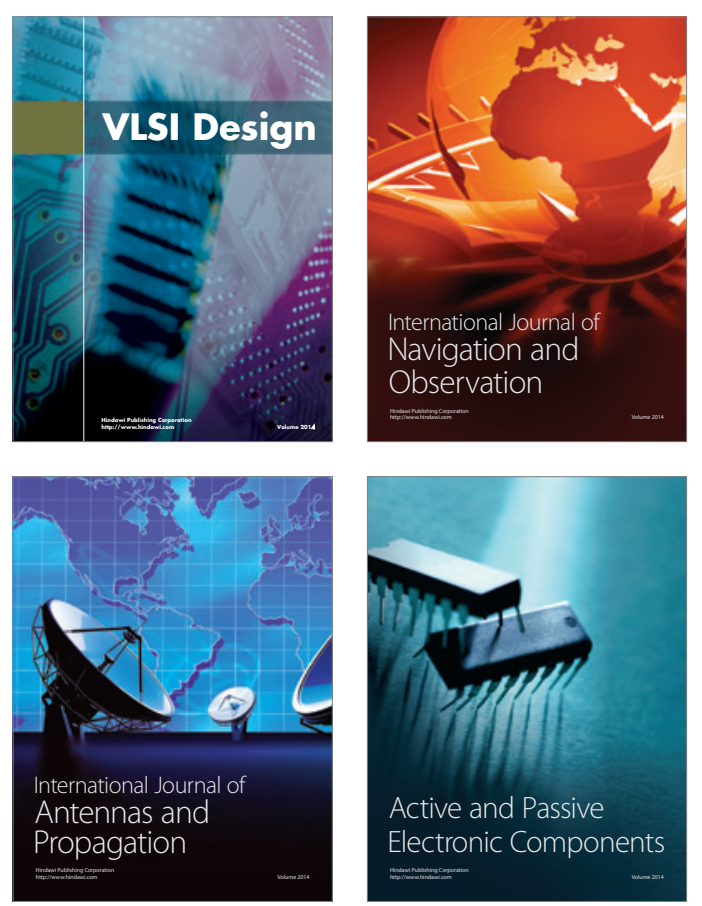
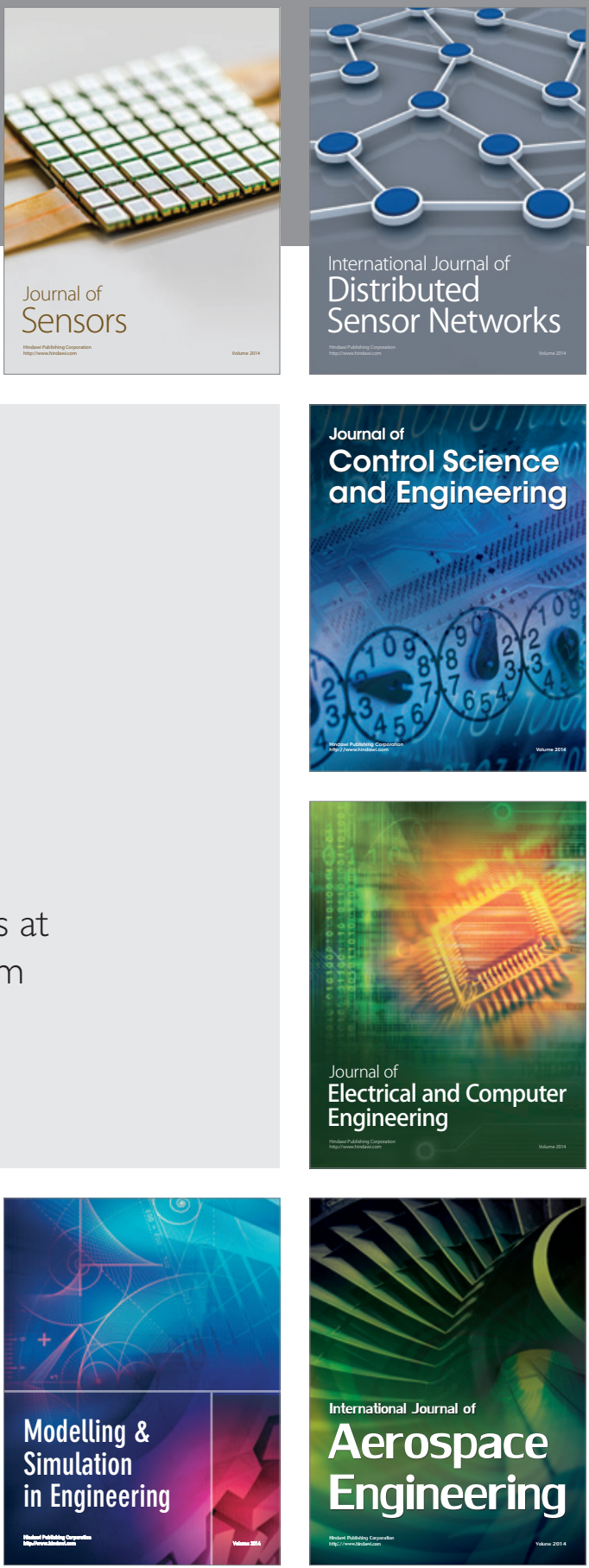

Journal of

Control Science

and Engineering
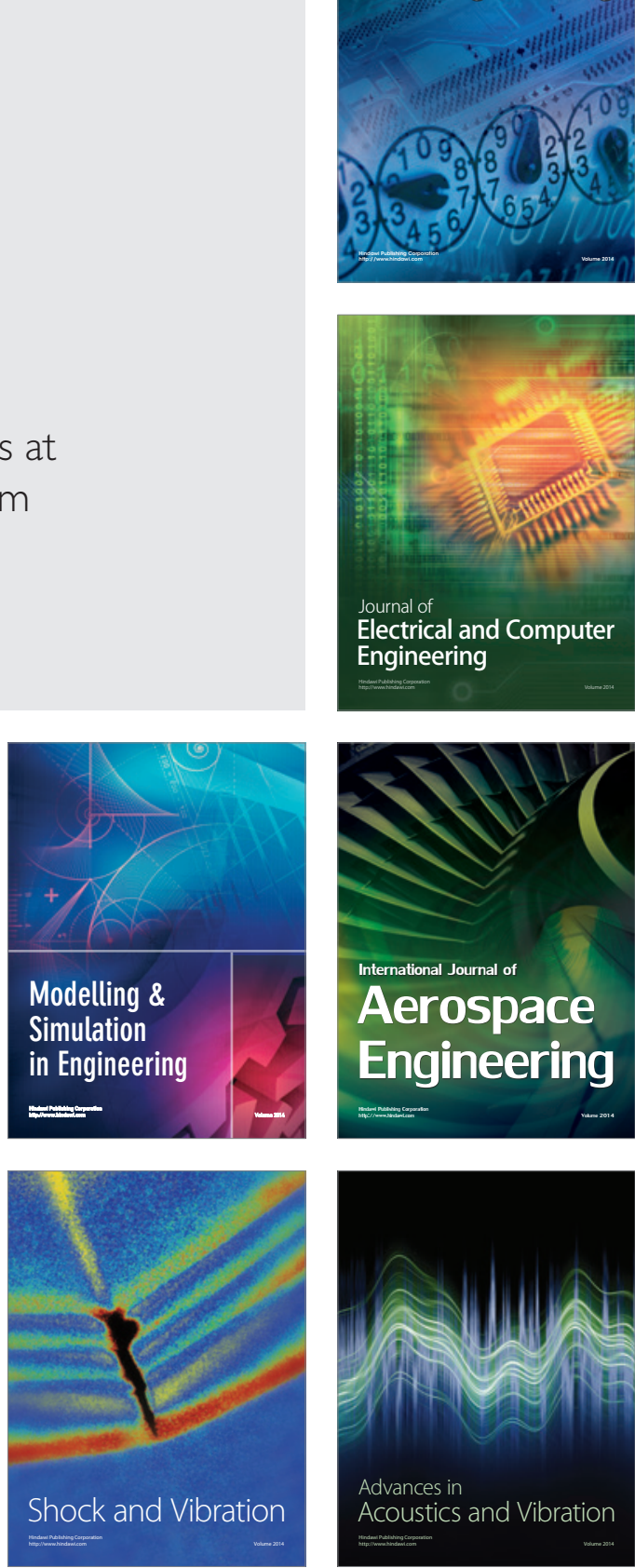